allele acquires from the degenerating sister nuclei a collection of chromatin fragments that are presumably heterochromatinized and silenced; they might also be covalently modified and therefore hard to detect by Southern blotting or by amplification in the polymerase chain reaction.

I propose that these supernumerary chromatin fragments propagate within the meristem cells of succeeding generations, and so are present in a very few cells of the plant; nevertheless, they are often present in the germ lines that are themselves derived from the meristem. Within a second-generation descendant that is homozygous for a chromosomal allele, such supernumerary chromatin fragments might harbour another allele in a cryptic state. These supernumerary fragments might pair with normal chromosomes in the male germ line preferentially, as indicated by Lolle et al. ${ }^{1}$, where they direct gene conversion of the chromosomal alleles. The gene-converted allele, therefore, would reappear as a mendelian factor in the third or a subsequent generation.

This proposal dispenses with the hypothetical RNA cache ${ }^{1}$, for which little evidence exists. It is consistent with the incidence of supernumerary chromosome fragments in plants, particularly with their known effects on pollen function ${ }^{3}$, and explains the lack of somatic convertant sector in any generation ${ }^{1}$; it also invokes standard molecular processes of DNA-directed gene conversion over relatively long regions of homology. The differences in the frequency of conversion of the three tested alleles ${ }^{1}$ may reflect co-conversion polarity ${ }^{4}$.

My proposal can also explain the curiously high frequency of allele reappearance in dissected embryos, which is roughly twice that found in mature plants (it increased from 10\% to $20 \%$ ). Assume that gene conversion produces a heteroduplex DNA that escapes mismatch repair. If conversion occurs in the haploid generative nucleus of the pollen, the two sperm cells will be non-identical at the converted allele. After double fertilization, the embryo and the endosperm will contain two different alleles. An embryo dissected from such a seed will inevitably be associated with endosperm cells, and will yield a signal in the polymerase chain reaction that is indistinguishable from a heterozygous embryo, although the embryo itself is homozygous for the allele. As this will occur as often as a conversion, the frequency of the total converted allele in dissected embryos and endosperms together should be about $20 \%$, an estimate close to the experimentally observed rate.

The model proposed here, but not one in which gene conversion is directed by $\mathrm{RNA}^{5}$ or by very short, dispersed, repeated DNA sequences ${ }^{6}$, is easily reconciled with the notion of co-conversion polarity ${ }^{4}$. It should be possible to test whether co-conversion polarity is a factor in the phenomenon revealed by Lolle et al. ${ }^{1}$ by producing double- and triple- mutant alleles through exploitation of this effect. Further investigations into the possibility and nature of DNA archiving in plants and of plant germ lines should prove interesting.

\section{Animesh Ray}

Keck Graduate Institute, Claremont, California 91711, USA

e-mail: aray@kgi.edu

1. Lolle, S. J., Victor, J. L., Young, J. M. \& Pruitt, R. E. Nature 434, 505-509 (2005)

2. Downs, D. M. \& Roth, J. R. Genetics 117, 367-380 (1987).

3. Stebbins, G. L. in Chromosomal Evolution in Higher Plants 67-71 (Addison-Wesley, Reading, Massachusetts, 1971).

4. Nicolas, A. \& Petes, T. D. Experientia 50, 242-262 (1994).

5. Baltimore, D. Cell 40, 481-482 (1985).

6. Chaudhury, A. Nature doi:10.1038/nature04062 (2005).

doi:10.1038/nature04063

Reply: Lolle et al. reply to this communication

(doi:10.1038/nature04064)

\title{
PLANT GENETICS
}

\section{Lolle et al. reply}

Reply to: A. Chaudhury (doi:10.1038/nature04062) and A. Ray (doi:10.1038/nature04063)

Chaudhury ${ }^{1}$ and $\mathrm{Ray}^{2}$ propose alternative models to account for our observed pattern of non-mendelian inheritance in the hothead (hth) mutant of Arabidopsis ${ }^{3}$.

Chaudhury suggests that the information required to restore correct genetic sequences in $h$ th mutant plants could be stored in short stretches of nucleotide sequence within the genome ${ }^{1}$. Although the sequences required for restoration are indeed present in the genome, the length of similarity seen in the 'reverting sequences' identified by Chaudhury is barely greater than would be expected from random chance. An appropriate control for his in silico experiment would be to establish how many similar sequences (13-18 nucleotides in length, with a single nucleotide mismatch relative to the sequence in the parent plant) are present in the genome that could likewise introduce silent nucleotide substitutions into the $h$ th gene under the same conditions.

Should there be a significant number of these sequences (and given that no such silent mutations occur in the corrected alleles ${ }^{3}$ ), then an explanation is needed for why some of them are used for correction whereas the majority are not. Even if silent mutation events occur independently of the reversion of the $h$ th mutation, we should still detect them in our small sample of sequenced reverted alleles owing to the relatively large number of possible silent mutations.

Chaudhury also suggests that the increased permeability of mutant $h$ th female gametophytes could allow DNA or RNA from the degenerating non-functional megaspores to enter the functional $h$ th megaspore and then be archived and carried forward to allow gene conversion in the next generation. This may be a possibility, but our previous work examined only changes in the permeability of the extracellular cuticle covering the outside of the epidermal cell layer ${ }^{4}$. We have no data concerning increased cellular permeability in $h$ th mutants. The fact that we see no obvious drop in the rate of reversion over several generations ${ }^{3}$ is inconsistent with Chaudhury's suggestion that this could be a second mechanism to bolster the rate of reversion for only a single generation.

Ray $^{2}$ claims that our results could be explained by the stable inheritance of supernumerary chromosomal fragments that are archived in a way that makes them inaccessible to DNA hybridization and the polymerase chain reaction. These fragments might also be restricted to meristematic cells and therefore be present in such low concentrations that they are undetectable in a conventional experiment. This is an interesting possibility that is consistent with our observations, but it postu- lates a novel system of segregation to restrict the chromosome fragments to what would constitute a hitherto undetected germ line in plants. Considering also Ray's explanation for the doubled rate of conversion in embryos, we note that it would be necessary for all conversion events to take place in the generative cell and to fail to be corrected by mismatch repair.

In summary, we agree with Ray that there is little direct evidence to support any given molecular identity for the cryptic templates that allow genetic restoration in $h t h$ mutant plants. We proposed that the templates might be a replicating form of RNA, but the data are also consistent with a form of DNA that is segregated into a limited number of cells in the plant or that is not readily detectable by conventional molecular techniques. This sequence archive (whether DNA or RNA) would therefore require the same basic properties as those we proposed ${ }^{3}$ : it would need to be replicated, transmitted with high fidelity over several generations, and retain the ability to restore nuclear DNA sequences.

Susan J. Lolle, Jennifer L. Victor, Jessica M. Young, Robert E. Pruitt

Department of Botany and Plant Pathology,

Purdue University, West Lafayette,

Indiana 47907-2054, USA

e-mail:pruittr@purdue.edu

1. Chaudhury, A. Nature doi:10.1038/nature04062 (2005).

2. Ray, A. Nature doi:10.1038/nature04063 (2005)

3. Lolle, S. J., Victor, J. L., Young, J. M. \& Pruitt, R. E. Nature 434, 505-509 (2005).

4. Lolle, S. J., Hsu, W. \& Pruitt, R. E. Genetics 149, 607-619 (1998).

doi:10.1038/nature04064 